\title{
Was erzählt Fritz Kreislers Geige?
}

\author{
Matej Santi, Wien
}

DOI: $10.25366 / 2020.109$

Zitation: Matej Santi, „Was erzählt Fritz Kreislers Geige?”, in: Brückenschläge zwischen Musikwissenschaft und Informatik. Theoretische und praktische Aspekte der Kooperation, in Verbindung mit der Fachgruppe Digitale Musikwissenschaft hrsg. von Stefanie Acquavella-Rauch, Andreas Münzmay und Joachim Veit (= Musikwissenschaft: Aktuelle Perspektiven. Bericht über die Jahrestagung der Gesellschaft für Musikforschung 2019 in Paderborn und Detmold, Bd. 3), Detmold, Musikwissenschaftliches Seminar der Universität Paderborn und der Hochschule für Musik Detmold, 2020, S. 207-210, DOI: 10.25366/2020.109 


\title{
Was erzählt Fritz Kreislers Geige?
}

\author{
MATEJ SANTI, WIEN
}

In diesem kurzen Beitrag wird der Bedeutung von audiovisuellen Quellen für die Erforschung von historisch gewachsenen Topoi - wie der "Wiener" Klanglichkeit - und deren Mitgestaltern - etwa dem Geiger Fritz Kreisler (1875-1962) - nachgegangen. Einige dieser Quellen wurden auf Onlineportalen digitaler Archive dank bereits vorhandener Metadaten recherchiert. Andere, bei denen bestimmte Akteur*innen, Orte oder Musik(stücke) nicht aus den vorhandenen Metadaten eruierbar waren, gerieten durch weiterführende Recherche ins Blickfeld. Daraus ergibt sich die wichtige Frage, ob solche nicht adäquat mit Metadaten erschlossene Quellen extern (das heißt nicht im Archiv, in dem sie gespeichert sind) mit Hilfe eines digitalen Tools durch weitere Metadaten angereicht werden könnten. Solche Metadaten würden nämlich die Auffindbarkeit von eventuell relevanten audiovisuellen Quellen im Rahmen weiterer Forschungsarbeiten wesentlich erleichtern.

Eine der jüngsten Quellen, die in der Österreichischen Mediathek aufbewahrt sind und sich um den Themenkomplex "Fritz Kreisler" drehen, ist die Videodokumentation der Restaurierungsarbeiten am Salonwagen der Kaiserin Elisabeth, der heute im Technischen Museum Wien ausgestellt ist. ${ }^{1}$ Die Aufnahme aus dem Jahr 2004 ist im Online-Archiv der Institution verfügbar und mit Fritz Kreislers Charakterstück Schön Rosmarin unterlegt. Dieses und weitere Charakterstücke komponierte Kreisler im ersten Jahrzehnt des 20. Jahrhunderts und schrieb sie Komponisten der Vergangenheit zu. Erst 1935 eröffnete er, dass er sie selbst verfasst hatte. ${ }^{2} \mathrm{Er}$ komponierte auch die Operetten Apfelblüten (1919) und Sissy (1932), Vorlage für die amerikanische Filmproduktion der Columbia Pictures The King Steps Out aus dem Jahr 1936, in der die von Kreisler komponierten Charakterstücke reichlich Verwendung fanden. ${ }^{3}$ Damit wurde ein wesentlich über Kreislers Musik vermitteltes Altösterreich-Bild produziert.

Fritz Kreisler war allerdings im angelsächsischen Sprachraum nicht nur als Geiger und Komponist bekannt. Seine Schrift Four Weeks in the Trenches, in der er bereits 1915 seine vierwöchige Erfahrung als Offizier der K.-u.-k.-Monarchie an der Front in Galizien schilderte, galt als eine der bedeutendsten Quellen für die Mentalitätsgeschichte im Ersten Weltkrieg - auf archive.org

1 Videodokumentation - Restaurierung des Salonwagens von Kaiserin Elisabeth [Ausschnitt], Österreichische Mediathek, <https://www.mediathek.at/atom/1596D2CA-084-000A6-00000E84-15961056> (24.08.2020).

2 Boris Schwarz, Art. "Kreisler, Fritz”, in: Grove Music Online, <https://doi.org/10.1093/gmo/9781561592630.article.15504>.

3 The King Steps Out, Regie: Josef von Sternberg, USA 1936; vgl. <https://www.imdb.com/title/tt0027847/> (24.08.2020). 
ist der englische Text sowohl als gedrucktes Buch als auch als Audiobuch abrufbar. ${ }^{4}$ Der Bericht Kreislers steht erst seit 2015 auch in der deutschen Übersetzung unter dem Titel Trotz des Tosens der Kanone zur Verfügung. ${ }^{5}$ Zur Einleitung der deutschen Übersetzung verfasste der Musikwissenschaftler und Geiger bei den Wiener Philharmonikern Clemens Hellsberg einen Aufsatz mit dem Titel Fritz Kreisler - Künstler und Humanist. Hellsberg schreibt sowohl Fritz Kreisler als auch Bruno Walter eine wesentliche Rolle in der Behauptung Wiens als "Welthauptstadt der Musik" zu. ${ }^{6}$ Bemerkenswerterweise erwähnt Hellsberg als Quintessenz des musikalischen Schaffens von Kreisler sein Caprice Viennois, das unter anderem auch als Signation der Reihe von Radiosendung Austria's Past is Üresent des englischsprachigen Senders Radio Österreich International im Jahr 1988 erklang. ${ }^{7}$

Weitere Quellen im Archiv der Österreichischen Mediathek, die auf Fritz Kreisler verweisen sind: die Verleihung des Karl-Renner-Preises an Kreisler im Jahr $1960^{8}$ und der Nachruf Bruno Walters auf Kreisler aus dem Jahr 1962. ${ }^{9}$ An die universalistischen Werte der Musik, auf die Hellsberg in seiner Einleitung zum Schrift Kreislers appelliert, bezog sich auch der Geiger Yehudi Menuhin in einer Aufnahme aus dem Jahr 1979, als der Kreisler-Violinwettbewerb zum ersten Mal in Wien stattfand. ${ }^{10}$ Diese Idee einer universalistischen und humanisierenden Wirkung der Musik stellte einen der zentralen Topoi dar, mit dem die Verbreitung der deutschen musikalischen Kultur im späten 19. und frühen 20. Jahrhundert vor allem in den USA assoziiert wurde. ${ }^{11}$

Die heute als "wienerisch" bezeichnete Klangkultur Kreislers wurde aber nicht von Anfang an mit Beifall aufgenommen. Sein durchgehendes Vibrato wurde zum Beispiel von einigen seiner Zeitgenossen als unangenehm und als Bruch mit der Tradition empfunden. Bedenkt man, dass Kreisler nur seine Jugend in Wien verbrachte, in Paris seine Ausbildung genoss, in New York und Berlin lebte und nach der Machtübernahme des NS-Regimes endgültig in die USA emigrierte (wie neben vielen anderen der bereits erwähnte Bruno Walter) erweist sich die oft

4 Fritz Kreisler, Four Weeks in the Trenches. The War Story of a Violinist, Boston 1915, <https://archive.org/details/ fourweeksintren00unkngoog/mode/2up>. LibriVox, <https://archive.org/details/four_weeks_trenches_0910_librivox> (beides 24.08.2020).

5 Fritz Kreisler, Trotz des Tosens der Kanone: Frontbericht eines Virtuosen, Wien 2015.

6 Clemens Hellsberg, „Fritz Kreisler - Künstler und Humanist”, in: Fritz Kreisler, Trotz des Tosens der Kanone: Frontbericht eines Virtuosen, Wien 2015, S. 8-19, hier S. 18.

7 Austria's Past is Present, Österreichische Mediathek, <https://www.mediathek.at/portalsuche/?q\%5B\%5D=Austria\%C2\%B4s+past+is+present> (24.08.2020).

8 Originalbericht von der Überreichung des Dr.-Karl-Renner-Preises an Fritz Kreisler durch den österreichischen Generalkonsul Dr. Karl Wolf und der anschließenden Rede von Fritz Kreisler, Österreichische Mediathek, <https:// www.mediathek.at/atom/09108FBC-244-00052-00000314-090FC364> (24.08.2020).

9 Nachruf von Bruno Walter auf Fritz Kreisler, Österreichische Mediathek, <https://www.mediathek.at/ atom/09186A43-320-00111-0000063C-0917AC63> (24.08.2020).

10 Von Tag zu Tag. Der Fritz-Kreisler-Wettbewerb, Österreichische Mediathek, <https://www.mediathek.at/ atom/0E68E11A-374-00034-0005BCB8-0E68387F> (24.08.2020).

11 Vgl. Jessica C. E. Gienow-Hecht, Sound Diplomacy: Music and Emotions in Transatlantic Relations (1850-1920), Chicago u. a. 2009, S. 20-39. 
vertretene Idee, dass sich "Heimat" erst im Exil herauskristallisiert, auch hier bestätigt. Das Narrativ von Kreisler als pars pro toto einer als wienerisch apostrophierten Klanglichkeit ist nichtdestotrotz aktueller denn je: 2018 wurde das Institut für Konzertfach (Streichinstrumente, Gitarre und Harfe) an der Universität für Musik und darstellende Kunst Wien nach Kreisler benannt. ${ }^{12}$

Bei der Quellensuche kamen verschiedene Strategien zum Einsatz. In einigen Fällen war das Stichwort "Kreisler" in den bereits bestehenden Metadaten, meistens in der Beschreibung des Dokumentes, vorhanden. Dies war bei der Recherche im Onlineportal der Österreichischen Mediathek der Fall. In anderen Fällen, wie bei der Signation der Radiosendung Austria's past is present, fehlte jegliche Referenz auf Kreisler - das Dokument wurde durch Zufall gefunden. Besonders solche Fälle machen deutlich, dass nur durch das Anreichern mit Metadaten die Präsenz von Musik(en) in unterschiedlichen audiovisuellen Kontexten sichtbar gemacht werden kann. Der Text Kreislers auf archive.org wurde durch eine Google-Recherche aufgespürt.

In archivierten audiovisuellen Dokumenten kommen aber auch weitere Entitäten wie Orte, Personen, Institutionen, Repertoires und Zeiten vor, die in den vorhandenen Metadaten nicht vertreten sind. Das Ziel eines externen digitalen Tools bestünde darin, die Netzwerke zwischen solche Entitäten sichtbar zu machen. Erst dadurch kann die Aufmerksamkeit auf die Schichten der Sedimentierung von Bedeutungszuschreibungen gelenkt werden. Nicht mehr die Darstellung von Chronologie, sondern die Kontinuitäten im Sinne von "longue durée" würden ins Zentrum des Interesses rücken.

Zitation: Matej Santi, „Was erzählt Fritz Kreislers Geige?", in: Brückenschläge zwischen Musikwissenschaft und Informatik. Theoretische und praktische Aspekte der Kooperation, in Verbindung mit der Fachgruppe Digitale Musikwissenschaft hrsg. von Stefanie Acquavella-Rauch, Andreas Münzmay und Joachim Veit (= Musikwissenschaft: Aktuelle Perspektiven. Bericht über die Jahrestagung der Gesellschaft für Musikforschung 2019 in Paderborn und Detmold, Bd. 3), Detmold, Musikwissenschaftliches Seminar der Universität Paderborn und der Hochschule für Musik Detmold, 2020, S. 207-210, DOI: 10.25366/2020.109

12 Universität für Musik und darstellende Kunst Wien, Fritz Kreisler Institut für Konzertfach Streichinstrumente, Gitarre und Harfe, <https://www.mdw.ac.at/str/> (24.08.2020). 


\section{Abstract}

This short contribution shows the relevance of audiovisual sources for the history of $20^{\text {th }}$ century music. It traces the role played by the violinist Fritz Kreisler (1875-1962) in shaping the widespread cliché of the "Viennese sound" via an examination of audiovisual sources. The sources stored in different online archives or social media portals play a key role, but the traceability of a given agent is not guaranteed. For this reason, controlled vocabularies and a digital tool which enable the addition of new metadata to already existing sources should be developed in the near future. This would enable researchers to trace agents, such as institutions and artists, and to connect them with places, repertoires and cultural topoi.

\section{Kurzvita}

Matej Santi studierte Geige und Musikwissenschaft. Am Institut für Musikwissenschaft und Interpretationsforschung (IMI) der Universität für Musik und darstellende Kunst Wien (mdw) schloss er das PhD-Studium mit einer Dissertation über die Rolle der Musik in der Ausbildung nationaler Identitäten in Triest ab. Derzeit ist er als Post-Doc am Institut für Musikwissenschaft und Interpretationsforschung beschäftigt (Projekt: Telling Sounds) und Lehrbeauftragter an der mdw sowie der Webster University Vienna. 



\section{Brückenschläge zwischen Musikwissenschaft und Informatik}

Theoretische und praktische Aspekte der Kooperation

Herausgegeben von Stefanie Acquavella-Rauch,

Andreas Münzmay und Joachim Veit 
Brückenschläge zwischen Musikwissenschaft und Informatik 


\section{Musikwissenschaft: Aktuelle Perspektiven}

Bericht über die Jahrestagung der Gesellschaft für Musikforschung 2019 in Paderborn und Detmold

Herausgegeben von Rebecca Grotjahn und Nina Jaeschke

Band 3 


\section{Brückenschläge zwischen Musikwissenschaft und Informatik}

Theoretische und praktische Aspekte der Kooperation

Beiträge der Symposien zur Digitalen Musikwissenschaft

Osnabrück 2018 und Paderborn 2019

im Rahmen der Jahrestagungen der Gesellschaft für Musikforschung

In Verbindung mit der Fachgruppe Digitale Musikwissenschaft herausgegeben von

Stefanie Acquavella-Rauch, Andreas Münzmay und Joachim Veit

Detmold: Musikwissenschaftliches Seminar der Universität Paderborn und der Hochschule für Musik Detmold 2020 
DOI: $10.25366 / 2020.87$

Online-Version verfügbar unter der Lizenz: Urheberrecht 1.0, $<$ https://rightsstatements.org/page/InC/1.0/?language =de >

Bibliografische Information der Deutschen Nationalbibliothek

Die Deutsche Nationalbibliothek verzeichnet diese Publikation in der Deutschen Nationalbibliografie; detaillierte bibliografische Daten sind im Internet über http://dnb.d-nb.de abrufbar.

\section{Impressum}

Redaktion: Stefanie Acquavella-Rauch, Andreas Münzmay und Joachim Veit Satz: Nina Jaeschke und Joachim Veit

(C) Musikwissenschaftliches Seminar der Universität Paderborn und der Hochschule für Musik Detmold 2020 


\section{INHALT}

Rebecca Grotjahn, Nina Jaeschke

Vorwort zu Band 1-3

IX

Stefanie Acquavella-Rauch, Andreas Münzmay, Joachim Veit

Brückenschläge zwischen Musikwissenschaft und Informatik - Vorbemerkung

$\mathrm{XI}$

\section{KOLLABORATIONEN - KO-LABORATORIEN}

\section{Reinhard Keil}

Der Computer als Denkzeug für hermeneutische Arbeit

\section{Ulrich Konrad}

Philologie und Digitalität. Perspektiven für die Musikwissenschaft im Kontext fächerübergreifender Institutionen

\section{Gudrun Oevel}

Infrastruktureinrichtungen in Forschungsprojekten - Spagat oder Chance?

\section{Dennis Ried}

Erhebung, Transformation und Präsentation digitaler Forschungsdaten

Anna Neovesky, Frederic von Vlahovits

IncipitSearch - Leitfaden zur Zusammenarbeit

Elisabeth Treydte

Clara Schumann \#digital. 40 Jahre Archiv Frau und Musik und der Start in die Digitalisierung

\section{TEXT/DATEN/PROZESSE}

\section{Christine Siegert}

Komponisten-Gesamtausgaben im digitalen Zeitalter: Perspektiven

und Reflexionen am Beispiel Ludwig van Beethovens

Markus Neuwirth, Johannes Hentschel, Martin Rohrmeier

Perspectives of Musical Corpus Studies: The Annotated Mozart Sonatas

Agnes Amminger, Franz Kelnreiter

Leopold Mozarts „Gründliche Violinschule". Zur Textcodierung und -präsentation einer digitalen Edition

\section{Oleksii Sapov}

Algorithmische Automatisierung komplexer Notationsregeln in MEI-XML am Beispiel von Versetzungszeichen 


\section{Susanne Cox, Richard Sänger}

Digitale Fassungsvergleiche am Beispiel von Beethovens Eigenbearbeitungen

Agnes Seipelt

Digitale Edition und Harmonische Analyse mit MEI von Anton Bruckners

Studienbuch

Stefanie Acquavella-Rauch

Musikalische Schaffensprozesse 2.0 - Inkorporation audiovisueller Medien

der populären Musik in Methoden der digitalen Edition

\section{DIGITAL(ISIERT)E MATERIALITÄTEN}

\section{Miriam Akkermann}

(Musik)Instrument (im) Computer

\section{Daniel Fütterer}

Herausforderungen bei der Kodierung von Paratext am Beispiel Neuer Musik mit Live-Elektronik

\section{Matthias Pasdzierny}

How much is the glitch? Das digitale Paradigma als Herausforderung

und Chance für die historische Musikwissenschaft

\section{Shintaro Miyazaki}

Musik für Maschinen?! - Wo sich die Wissenschaft der Medien, des Computers und der Musik treffen und wie sie zusammenarbeiten könnten

\section{MUSIKGESCHICHTE(N) IM NETZ}

\section{Matthias Tischer}

Musikgeschichte der DDR: Ein Pilotprojekt zur digitalen Musikvermittlung

Annette van Dyck-Hemming, Jan Eberhardt, Melanie Wald-Fuhrmann

Ansätze zur Analyse historischer Netzwerke mit Neo4j® - Aus der Projekt-Werkstatt der Datenbank zur Fachgeschichte der Musikwissenschaft

Axel Beer, Martin Bierwisch, Kristina Krämer

Das MMM2 - Ein regionalgeschichtliches Onlinelexikon der Arbeitsgemeinschaft für mittelrheinische Musikgeschichte

\section{Matej Santi}

Was erzählt Fritz Kreislers Geige?

\section{Elias Berner}

Alle Menschen werden Brüder?! Ein historisches Dokument aus dem

Nationalsozialismus in den sozialen Medien 
Gabriele Buschmeier in memoriam 